\title{
Adsorção de cromo (III) por resíduos de laranja in natura e quimicamente modificados
}

\section{Adsorption of chromium (III) by waste orange raw and chemically modified}

\author{
João Valdir Tadioto Miranda de Souza ${ }^{1}$; Cristina Lorena Massocatto ${ }^{2}$; Kristiany \\ Moreira Diniz ${ }^{3}$; César Ricardo Teixeira Tarley4; Josiane Caetano ${ }^{5}$; Douglas \\ Cardoso Dragunski ${ }^{6}$.
}

\section{Resumo}

Os rejeitos contendo metais pesados, originados de atividades industriais e mineradoras, podem ocasionar danos ao meio ambiente e à saúde humana. Mesmo o cromo, considerado um metal essencial, quando encontrado em altas concentrações, pode ser prejudicial, razão pela qual, o objetivo deste trabalho foi investigar a eficiência de tratamentos químicos em casca, bagaço e casca com bagaço de laranja, com o intuito de tratar efluentes aquosos contaminados por cromo (III). A modificação referese a alteração da estrutura química dos resíduos de laranja com hidróxido de sódio e ácido cítrico. Foram realizados ensaios em sistemas de batelada contendo cromo, sendo que as concentrações foram determinadas através da utilização de um espectrofotômetro de absorção atômica com chama. Analisouse a adsorção em função do $\mathrm{pH}$, tempo de contato, concentração do metal, capacidade de dessorção e fatores termodinâmicos. As modificações foram constatadas no infravermelho pelo surgimento de um pico em $1730 \mathrm{~cm}^{-1}$, o qual se refere aos grupos carboxilatos. O tempo necessário para o sistema de adsorção atingir o equilíbrio foi de cerca de 500 minutos e a cinética segue um comportamento descrito pela equação de pseudo-segunda ordem. Avaliou-se a adsorção em relação aos modelos de Langmuir e Freundlich, sendo o modelo de Freundlich o que se aplicou melhor ao processo. O valor da capacidade máxima de adsorção foi de $75,64 \mathrm{mg} . \mathrm{g}^{-1}$ para a casca modificada com $\mathrm{NaOH}$. Além de aumentar as propriedades adsorventes, o sistema tornou-se mais espontâneo energeticamente após o tratamento químico, verificado pelos baixos valores da energia de Gibbs.

Palavras-chave: Adsorção. Cromo. Resíduos de laranja. Modificação química.

\section{Abstract}

The waste containing heavy metals originating from mining and industrial activities, can cause damage to the environment and human health. Although chromium is considered an essential metal, when found

\footnotetext{
${ }^{1}$ Graduado em Química e Mestre em Biotecnologia Aplicada à Agricultura, Universidade Paranaense.

${ }^{2}$ Graduada em Farmácia e aluna de mestrado em Biotecnologia Aplicada à Agricultura, Universidade Paranaense.

3 Graduada em Química e aluna de mestrado em Química, Universidade Estadual de Londrina.

${ }^{4}$ Docente do Curso de Química, Universidade Estadual de Londrina.

5 Docente do Curso de Química, Universidade Estadual do Oeste do Paraná.

${ }^{6}$ Docente do mestrado em Biotecnologia Aplicada à Agricultura da Universidade Paranaense; dcdragunski@unipar.br.
} 
in high concentrations can be harmful. Therefore, the aim of this study was to investigate the efficiency of chemical treatments in husk, bagasse and bark with orange pulp, in order to treat wastewater aqueous contaminated by chromium (III). The modification refers to a treatment sodium hydroxide and citric acid, which aims at introducing carboxylate groups. Tests were conducted in batch systems containing chromium, and the concentrations were determined using the atomic absorption spectrophotometer flame. We analyzed the adsorption as a function of $\mathrm{pH}$, contact time, metal concentration, the desorption capacity and thermodynamic parameters. The changes were observed in the infrared by the appearance of a peak at $1730 \mathrm{~cm}^{-1}$, which refers to carboxylate groups. The time required to reach the adsorption system balance was approximately 500 minutes and the kinetics follows a behavior described by the equation of pseudo-second order. Was evaluated in relation to the adsorption models of Langmuir and Freundlich, being the model of Freundlich the best applied to the process. Besides increasing the adsorbent properties, the system became more spontaneous after the chemical treatment, verified by the low values of Gibbs energy.

Key words: Adsorption. Chrome. Orange waste. Chemical modification.

\section{Introdução}

Os rejeitos contendo metais pesados, originados de atividades industriais e mineradoras, podem ocasionar danos ao meio ambiente e à saúde humana (YAMAMURA; YAMAURA, 2005). Mesmo o cromo (Cr), considerado um metal essencial, quando encontrado em altas concentrações, pode ser prejudicial à vida humana, à fauna e à flora. Em virtude disso, a resolução CONAMA (Conselho Nacional do Meio Ambiente) 357/2005, estabelece que a concentração máxima encontrada deste metal em água doce seja de $0,05 \mathrm{mg} \mathrm{L}^{-1}$.

$\mathrm{O} \mathrm{Cr}$ existe no meio ambiente principalmente na sua forma hexavalente (VI) e trivalente (III). Embora se saiba que o $\mathrm{Cr}$ (VI) é mais tóxico que o Cr (III), a exposição prolongada ao $\mathrm{Cr}$ (III) também pode causar problemas como alergias na pele e câncer em seres humanos (YUN et al. 2001). Além disso, o Cr (III) pode sob certas condições ser oxidado para o estado mais carcinogênico e mutagênico Cr (VI), por algumas bactérias ou pelo óxido de manganês presente no ambiente (SETHUNATHAN et al., 2005).

Existem diversos métodos convencionais de tratamento de efluentes contendo metais pesados como precipitação, oxidação ou redução, filtração, troca iônica, tratamento eletroquímico, dentre outros. Porém, muitas vezes são inviáveis por diversos problemas técnicos, custos de operação e dos materiais utilizados (COSTA; SCHNEIDER; RÚBIO, 1999).

A adsorção éum método alternativo de tratamento utilizado para a remoção de metais tóxicos de efluentes industriais. Nesse fenômeno, é chamado de adsorvente o material sobre o qual ocorre a adsorção, e de adsorbato a substância adsorvida (PORPINO, 2009). O emprego dessa técnica tem sido considerado superior a outras técnicas para reuso de água em termos de custo inicial, flexibilidade, simplicidade de projetos e facilidade de operação. Contudo, para que o processo de adsorção seja eficiente, alguns critérios devem ser obedecidos na escolha de um adsorvente, pois este deve apresentar alta seletividade, alta capacidade, longa vida, baixo custo e estar disponível em grandes quantidades (IMMICH, 2006).

Devido a procura de materiais que ofereçam um bom desempenho associado a um baixo custo, a busca desses adsorventes está focada nas biomassas constituídas de resíduos naturais. O processo de biossorção baseia-se na propriedade desses adsorventes em reter íons de soluções aquosas, sendo por isso, considerados promissores na remoção de metais pesados de efluentes industriais (PAGNANELLI et al., 2001). 
A biossorção é resultado da interação eletrostática e também da formação de complexos entre íons metálicos e grupos funcionais presentes nas biomassas, quando estes possuem afinidade química pelo metal (RODRIGUES et al., 2006). Devido a isto, modificações químicas que possuem a finalidade de introduzir grupos funcionais na estrutura desses adsorventes, podem aumentar a capacidade de adsorção desses metais (SANTOS et al., 2010; SANTOS et al., 2011).

Existem resultados de pesquisas envolvendo diversas biomassas para remoção de poluentes aquosos, tais como: resíduos de castanha (YAO; QI; WANG, 2010), casca de amendoim (LIU; SUN; LI, 2010), casca de laranja (ANNADURAI; JUANG; LEE, 2003), (FENG; GUO; LIANG, 2009), (PÉREZ-MARÍN et al., 2007), casca de banana (ANNADURAI; JUANG; LEE, 2003), fungos (BALDISSERA, 2007), casca de arroz (MIMURA et al., 2010), bagaço de cana-de-açúcar (KARNITZ JÚNIOR et al., 2009), (KARNITZ JÚNIOR; GURGEL; GIL, 2010), (SANTOS et al., 2010), (SANTOS et al., 2011), dentre outros materiais celulósicos (KARNITZ JÚNIOR et al., 2009), (KARNITZ JÚNIOR; GURGEL; GIL, 2010).

Estudos recentes destacam a utilização de resíduos de laranja para a adsorção de metais devido à grande produção renovável e o baixo custo para estes adsorventes (KURNIAWAN et al., 2006). Esta biomassa apresenta grande capacidade de reter íons metálicos através da adsorção, sendo esta aumentada significativamente quando modificada quimicamente (MARSHALL et al., 1999).

O objetivo deste trabalho foi investigar a eficiência de tratamentos químicos dados aos resíduos de laranja, visando o tratamento de efluentes aquosos contaminados por metais, especialmente o Cr (III), avaliando-se a influência do $\mathrm{pH}$, tempo, concentração do metal presente, capacidade de dessorção e fatores termodinâmicos.

\section{Parte Experimental}

\section{Reagentes}

Todos os produtos utilizados no presente trabalho possuíam grau analítico. As soluções de $\mathrm{Cr}$, que simularam a presença do metal em um efluente industrial, foram preparadas com nitrato de cromo $\left(\mathrm{Cr}\left(\mathrm{NO}_{3}\right)_{3}\right.$ Vetec, 98\%), e as diluições foram realizadas para as concentrações desejadas. Soluções de $\mathrm{NaOH}$ (F. Maia, 97\%) 0,1 mol L-1 e HCl (F. Maia, 37\%) 0,1 mol L-1 e ácido cítrico 1,2 mol $\mathrm{L}^{-1}$ foram utilizadas para o controle do $\mathrm{pH}$ e para as modificações químicas na biomassa.

\section{Equipamentos}

Os espectros de Infravermelhos FTIR foram obtidos através do aparelho BOMEM, modelo MB-102. Macerou-se as amostras dos resíduos juntamente com os cristais de $\mathrm{KBr}$ numa proporção de $1 \mathrm{mg}$ amostra para $100 \mathrm{mg} \mathrm{KBr}$, e foram colocadas em um pastilhador. Analisou-se a faixa espectral de $4000-400 \mathrm{~cm}^{-1}$.

As amostras foram secas em estufa de circulação de ar e pesadas em balança analítica. Utilizou-se moinho analítico (QUIMIS) e agitador orbital (ITR). A quantificação do metal foi realizada utilizando um espectrofotômetro de absorção atômica em chama, modelo GBC 932plus.

\section{Modificação dos resíduos}

Para a realização dos experimentos foram utilizadas laranjas adquiridas de um supermercado, localizado em Umuarama - Paraná. Destas, retirouse o suco separando-as em casca (C), bagaço (B) e casca com bagaço (CB). Secou-se este material em estufa com circulação de ar até retirada da umidade e posteriormente triturou-se e peneirou-se (peneira Bertel, 100 mesh) a fim de se obter uma granulometria mais homogênea para a realização de duas modificações químicas. 


\section{Modificação com $\mathrm{NaOH}(\mathrm{S})$}

Para este procedimento, a cada grama do material foi adicionado $20 \mathrm{~mL}$ de uma solução de 0,1 mol L-1 NaOH. A mistura foi agitada por $2 \mathrm{~h}$ e o líquido descartado, posteriormente, o material foi lavado com água ultra-pura (Milli-Q) repetidas vezes e seco à $55^{\circ} \mathrm{C}$, durante $24 \mathrm{~h}$.

\section{Modificação com NaOH e Ácido Cítrico (SAC)}

Após ter recebido o tratamento com $\mathrm{NaOH}$, como já foi descrito, parte das amostras foram submetidas a modificação química com ácido cítrico. Para este procedimento, aos resíduos de laranja foi adicionado uma solução de $1,2 \mathrm{~mol} \mathrm{~L}^{-1}$ de ácido cítrico na proporção de $8,3 \mathrm{~mL}$ de solução por grama de amostra, onde permaneceu em agitação por 30 minutos e o líquido foi descartado. Todo o material foi lavado com água Milli-Q repetidas vezes e seco a $55^{\circ} \mathrm{C}$ durante $24 \mathrm{~h}$.

\section{Experimento de Adsorção dos Metais}

Adsorção em função do $\mathrm{pH}$

Realizaram-se as adsorções de Cr (III) em função do $\mathrm{pH}$ nos resíduos modificados e nos resíduos in natura, com a finalidade de obter-se o melhor valor de adsorção. Para realização deste experimento, agitou-se em agitador orbital por $24 \mathrm{~h}, 50 \mathrm{~mL}$ da solução de $\mathrm{Cr}$ em concentração inicial de $700 \mathrm{mg}$ $\mathrm{L}^{-1}$, em uma faixa de $\mathrm{pH}$ que variou de 2 à 6 . Com os dados obtidos calculou-se a quantidade do metal adsorvido por grama resíduo (qeq), utilizando-se a seguinte equação (1):

$$
q_{e q}=\frac{\left(c_{o}-c_{e q}\right) V}{M}
$$

Onde, $\mathrm{C}_{0}$ e $\mathrm{C}_{\mathrm{eq}}$ as concentrações iniciais e em equilíbrio do metal em solução $\left(\mathrm{mgL}^{-1}\right)$, respectivamente, $\mathrm{V}$ é o volume da solução em litro (L) e M a massa do resíduo em grama (g).
Adsorção em função do tempo

Foi realizada a adsorção do Cr na casca, bagaço e casca com bagaço modificados, bem como em todas estas amostras in natura, com o intuito de analisar suas propriedades adsorventes, sem qualquer tipo de lavagem ou tratamento. Desta forma, foi utilizado $50 \mathrm{~mL}$ da solução de $\mathrm{Cr}$ em concentração inicial de $700 \mathrm{mg} \mathrm{L}^{-1}$ para cada resíduo de laranja, onde esta foi agitada com $0,5 \mathrm{~g}$ de amostra em agitador orbital.

Foram retiradas dez alíquotas de $500 \mu \mathrm{L}$ em um intervalo de tempo de 15 a 1800 minutos, o volume foi aferido com água Milli-Q até $50 \mathrm{~mL}$. Em seguida as soluções foram analisadas em espectrofotômetro de absorção atômica com chama para se determinar as concentrações em cada coleta. Em cada alíquota foi realizada as correções em relação a quantidade de matéria. Ao final com os dados obtidos foi calculada a quantidade do metal adsorvido por grama de amostra $\left(\mathrm{q}_{\mathrm{eq}}\right)$ em função do tempo.

\section{Isotermas de adsorção}

Preparou-se soluções de $\mathrm{Cr}$, em diferentes concentrações, que variaram entre 100 e $1000 \mathrm{mg}$ $\mathrm{L}^{-1}$. Para a adsorção deste metal, foram utilizados $50 \mathrm{~mL}$ das soluções de $\mathrm{Cr}$, individualmente em $0,5 \mathrm{~g}$ de amostra de casca, bagaço e casca com bagaço in natura e modificado. Estas foram agitadas em agitador orbital durante $500 \mathrm{~min}$ e o $\mathrm{pH}$ foi mantido constante em 5 com a adição de $\mathrm{NaOH}$ ou $\mathrm{HCl}$. Em seguida as misturas foram filtradas e as soluções analisadas em espectrofotômetro de absorção atômica com chama para se determinar as concentrações iniciais e finais. Ao final, com os dados obtidos foi calculada a quantidade do metal adsorvido por grama de amostra, construindo um gráfico de qeq $\left(\mathrm{mg} \mathrm{g}^{-1}\right)$ em função de Ceq $\left(\mathrm{mg} \mathrm{L}^{-1}\right)$.

\section{Dessorção}

Para avaliar a capacidade de dessorção dos 
materiais estudados, após a realização da adsorção do cromo, os resíduos de laranja foram lavados com água Milli-Q e secos por $24 \mathrm{~h}$ em estufa a $50^{\circ} \mathrm{C}$. Posteriormente, foram colocados em $25 \mathrm{~mL}$ de solução de $\mathrm{HCl} 0,1 \mathrm{~mol} \mathrm{~L}^{-1}$, sob agitação constante por $24 \mathrm{~h}$ e temperatura $25 \mathrm{oC}$, para recuperação do metal. Analisou-se a concentração resultante em espectrofotômetro de absorção atômica com chama e verificou-se a porcentagem dessorvida.

\section{Adsorção em função da temperatura}

Os parâmetros termodinâmicos foram calculados por meio de experimentos de adsorção em função da temperatura. Desta forma, 0,5 $\mathrm{g}$ das amostras, correspondente aos resíduos de laranja in natura e modificados, foram agitados com $50 \mathrm{~mL}$ de solução a $700 \mathrm{mg} \mathrm{L}^{-1}$ de cromo, em pH $5 \mathrm{sob}$ diferentes temperaturas: $10^{\circ} \mathrm{C}, 20^{\circ} \mathrm{C}, 30^{\circ} \mathrm{C}, 40^{\circ} \mathrm{C}$ e $60^{\circ} \mathrm{C}$, durante $24 \mathrm{~h}$. As misturas foram filtradas e as soluções analisadas no espectrofotômetro de absorção atômica com chama.

\section{Resultados e discussão}

\section{Caracterização do adsorvente}

O fenômeno da adsorção ocorre devido à presença de grupos funcionais que constituem o material adsorvente. Desta forma, utilizou-se a técnica de espectroscopia na região do infravermelho para elucidar os grupos funcionais que podem estar presentes na estrutura do adsorvente. Picos em 3429, 2920, 1735, 1629, 1240 e 1050 cm$^{-1}$ são observados nos espectros representados na Figura 1a, 1b e 1c. A banda larga e forte em $3429 \mathrm{~cm}^{-1}$ é atribuído ao alongamento vibracinal da ligação O-H. O pico em $2920 \mathrm{~cm}^{-1}$ é referente ao alongamento vibracional da ligação C-H. Os picos em 1735 e $1629 \mathrm{~cm}-1$ podem ser atribuídos ao alongamento vibracional da ligação C-O. Segundo a literatura, o número de onda da absorção do grupo carbonila em compostos contendo grupamentos éster e carboxila é de aproximadamente $1735 \mathrm{~cm}^{-1}$ (TANA et al.,
2010). O pico em $1240 \mathrm{~cm}^{-1}$ podem ser atribuídos as vibrações da ligação C-O. As fortes bandas em $1050 \mathrm{~cm}-1$ confirmou a presença de polissacarídeo (TANA et al., 2010). Assim, pode-se concluir que existe hidroxila, carbonila e os grupos carboxílicos nos resíduos de laranja.

Figura 1 - Espectro de infravermelho dos resíduos de laranja. (A) Casca da laranja, (B) Bagaço da laranja, (C) Casca com bagaço. (a) in natura, (b) modificada com $\mathrm{NaOH}$, (c) modificada com $\mathrm{NaOH}$ e ácido cítrico
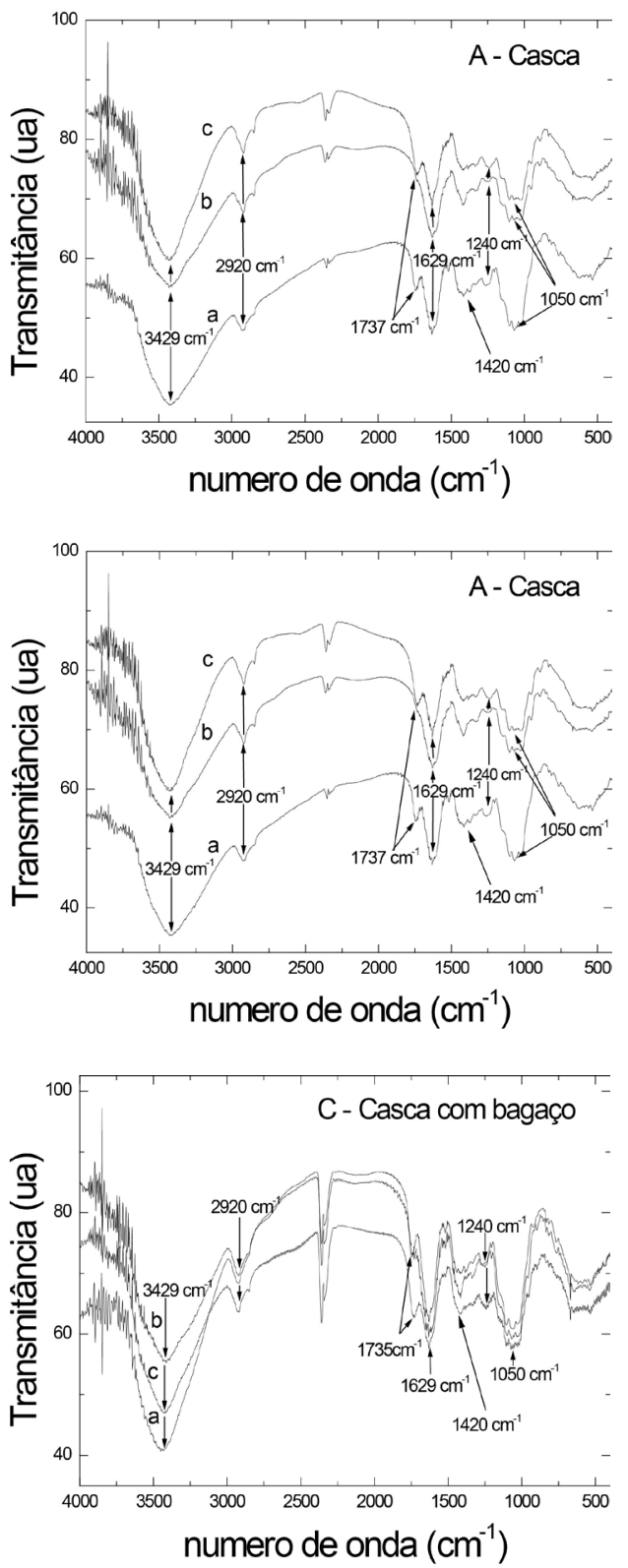

Fonte: Dados do autor. 
Ao realizar as modificações nos resíduos observou-se que houve uma alteração na intensidade do pico em $1735 \mathrm{~cm}^{-1}$, indicando um aumento na quantidade de grupamentos carboxila. Estes dados corroboram com o trabalho realizado por Rodrigues et al. (2006), que estudou a adsorção de metais em amostras de serragem de Paraju tratados quimicamente com $\mathrm{NaOH}$ e ácido cítrico. Estes grupos são importantes, pois com a variação do $\mathrm{pH}$ eles podem apresentar cargas negativas, contribuindo para uma maior interação com metais.

\section{Influência do $\mathrm{pH}$}

$\mathrm{O}$ pH da solução afeta a carga da superfície do adsorvente, o grau de ionização e especiação dos grupos de funções de superfície. Como grau de hidrólise de íons metálicos em solução aquosa é pHdependente, o pH de soluções influencia fortemente a especiação de íons metálicos (PEHLIVAN et al., 2009).

A adsorção de $\mathrm{Cr}$, em função do $\mathrm{pH}$, para resíduos de laranja estão apresentados na Figura 2. Constatou-se que o processo de adsorção é dependente do $\mathrm{pH}$, sendo que a capacidade de interação do $\mathrm{Cr}$ com a biomassa foi maior em valores de $\mathrm{pH}$ mais elevados. Isto pode ser explicado porque os grupos funcionais da biomassa são protonados, carregado positivamente, em $\mathrm{pH}$ mais baixo, não sendo favorável à adsorção do metal, porém, com o aumento do $\mathrm{pH}$ da solução, promoveu-se o desprotonamento destes grupos, deixando-os com carga negativa, aumentando a interação com íon de carga positiva. Portanto, ao analisar a Figura 2, observou-se que os maiores valores de adsorção para o $\mathrm{Cr}$ ocorreram em $\mathrm{pH}$ 5,0 ou 6,0 . Embora a adsorção do $\mathrm{Cr}$, para a alguns resíduos, tenha sido maior em $\mathrm{pH}$ 6,0, optou-se por utilizar o pH 5,0 em todos os estudos, para evitar uma possível precipitação de $\mathrm{Cr}$.
Figura 2 - Influência do $\mathrm{pH}$ na adsorção de $\mathrm{Cr}$ (III) nos resíduos de laranja: C, C-S, C-SAC, B, B-S, B-SAC, CB, CB-S, CB-SAC. Condições experimentais: $\mathrm{Cr}$ (III) $-700 \mathrm{mg} \mathrm{L}-1$, tempo de agitação $24 \mathrm{~h}$ e temperatura $250 \mathrm{C}$.

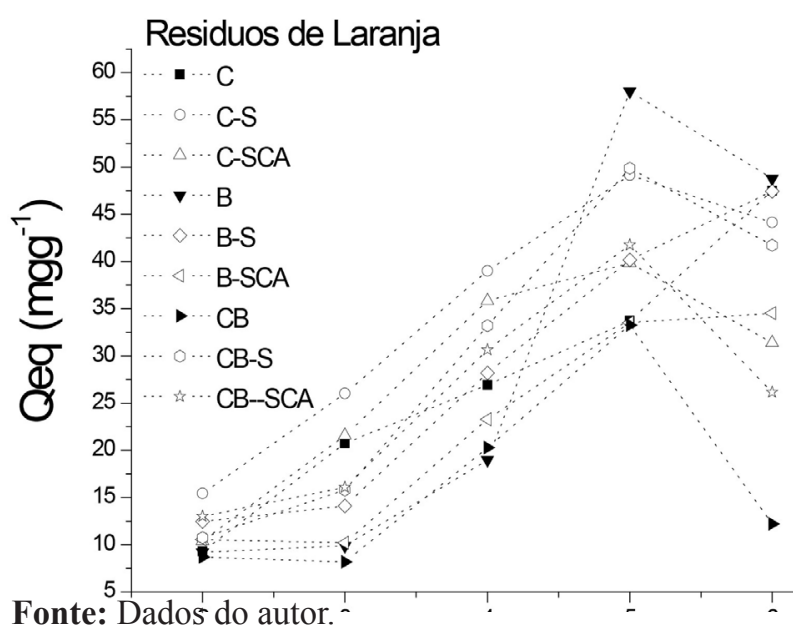

\section{Influência do tempo de adsorção}

A influência do tempo de adsorção na capacidade adsortiva de cromo nos materiais estudados é apresentada na Figura 3. Constatou-se que a partir de 500 minutos a adsorção se tornou praticamente constante. Este valor é considerado satisfatório quando comparado com outros adsorventes como o bagaço de cana, que teve uma estabilização a partir de 24h (SANTOS et al., 2010).

Pode-se observar que após as modificações com $\mathrm{NaOH}$ ocorreu um aumento significativo nas adsorções, comprovando a eficiência das mesmas em atrair o metal $\mathrm{Cr}$ (III).

Com os dados obtidos da cinética de cada adsorvente, pode-se aplicar os modelos de cinética de pseudo-primeira ordem e pseudo-segunda ordem, com a finalidade de se compreender melhor o mecanismo de sorção. A cinética de pseudoprimeira ordem segue o modelo de Lagergren, expressa pela equação 2 (DOĞAN et al., 2004), (NOELINE; MANOHAR; ANIRUDHAN, 2005), (ÖZCAN et al., 2005), (PÉREZ-MARÍN et al., 2007), (FENG et al. 2009). 


$$
\log \left(q_{e q}-q_{t}\right)=\log q_{e q}-\frac{\mathrm{K}_{1} \times t}{2,303}
$$

Onde, qt é a quantidade adsorvida de íons metálicos ( $\left.\mathrm{mg} \mathrm{g}^{-1}\right)$ no tempo $\mathrm{t}$ (min) e $\mathrm{K} 1$ é a constante de pseudo-primeira ordem $\left(\mathrm{min}^{-1}\right)$. Ao fazer o gráfico de $\log \left(\mathrm{q}_{\mathrm{eq}}-\mathrm{q}_{\mathrm{t}}\right)$ em função do tempo, obtiveram-se os valores de qeq e K1, coeficiente linear e angular, respectivamente. Podese observar (Tabela 1) que os valores de qeq obtidos experimentalmente não são próximos do calculado através da equação 2. Além disso, os valores de $r^{2}$ estão distantes de 1 , sugerindo que a adsorção não segue uma reação de primeira ordem. Com isto, aplicou-se o modelo de pseudo-segunda ordem (equação 3) para a cinética de adsorção do metal em diferentes resíduos de laranja (DOĞAN et al., 2004), (NOELINE; MANOHAR; ANIRUDHAN, 2005), (ÖZCAN et al., 2005), (PÉREZ-MARÍN et al., 2007), (FENG et al., 2009).

$$
\frac{t}{q_{t}}=\frac{1}{K_{2} q_{\mathrm{eq}}^{2}}+\frac{t}{q_{\mathrm{eq}}}
$$

onde $\mathrm{k}_{2}$ é a constante de pseudo-segunda ordem (g mg.-1min-1) obtida pelo coeficiente linear gráfico de $t / q_{t}$ em função de $t$. Também pode-se $o$ valor de qeq por meio do coeficiente angular. Os valores experimentais e calculados de $\mathrm{q}_{\mathrm{eq}}, \mathrm{k}_{2}$ e $\mathrm{r}^{2}$ estão expressos na tabela 1 .

Figura 3 - Cinética da adsorção do Cr (III) para os resíduos de laranja: C, C-S, C-SAC, B, B-S, B-SAC, CB, CB-S, CB-SAC. Condições experimentais: $\mathrm{pH}$ 5, Cr (III) - $700 \mathrm{mg} \mathrm{L-1}$ e temperatura 250C.

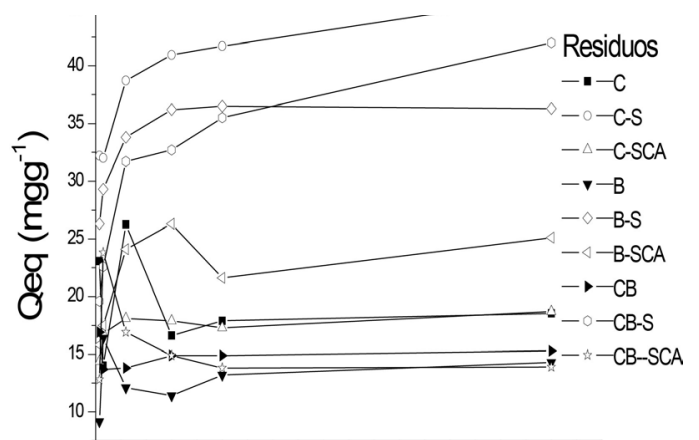

Os dados experimentais tiveram um melhor ajuste para o modelo de pseudo-segunda ordem por apresentar valores experimentais $\left(Q_{\text {eq }}\right.$ exp.) próximos do calculado pela equação 3 , além disso, os valores obtidos para $r^{2}$ foram próximos a 1 (SPINELLI et al., 2005).

Tabela 1 - Parâmetros para cinética nos modelos de pseuso-primeira e pseudo-segunda ordem para adsorção de $\mathrm{Cr}$ nos resíduos de laranja: C, C-S, C-SAC, B, B-S, B-SAC, CB, CB-S, CB-SAC.

\begin{tabular}{lccccccccc}
\hline & \multicolumn{3}{c}{ Cinética pseudo- } & \multicolumn{4}{c}{ Cinética pseudo- } \\
& \multicolumn{3}{c}{ primeira } & & \multicolumn{4}{c}{ segunda } \\
\multicolumn{1}{c}{$\mathbf{Q}_{\text {eq }}$} & $\mathbf{K}_{1}$ & $\mathbf{Q}_{\text {eq }}$ & $\mathbf{r}_{2}$ & $\mathbf{k}_{2}$ & $\mathbf{Q}_{\text {eq }}$ & $\mathbf{r}_{2}$ \\
Materiais & (exp.) & & (cal.) & & & (cal.) & \\
\hline C & 23,00 & $5,3 E-4$ & 7,53 & 0,33 & $1,4 \mathrm{E}-3$ & 23,30 & 0,99 \\
C(S) & 52,58 & $2,4 \mathrm{E}-3$ & 17,69 & 0,85 & $5,6 \mathrm{E}-4$ & 52,99 & 0,99 \\
C(SAC) & 20,44 & $2,5 \mathrm{E}-4$ & 9,88 & 0,53 & $1,1 \mathrm{E}-2$ & 20,75 & 0,99 \\
B & 20,43 & $5,3 \mathrm{E}-4$ & 12,90 & 0,76 & $9,9 \mathrm{E}-4$ & 21,11 & 0,98 \\
B(S) & 45,36 & $4,5 \mathrm{E}-3$ & 17,27 & 0,95 & $1,9 \mathrm{E}-4$ & 45,89 & 0,99 \\
B(SAC) & 22,51 & $2,2 \mathrm{E}-5$ & 4,76 & 0,03 & $4,0 \mathrm{E}-3$ & 22,54 & 0,99 \\
CB & 19,90 & $3,2 \mathrm{E}-4$ & 12,36 & 0,68 & $9,7 \mathrm{E}-4$ & 19,83 & 0,98 \\
CB(S) & 40,62 & $1,4 \mathrm{E}-3$ & 12,25 & 0,71 & $9,3 \mathrm{E}-4$ & 41,22 & 0,99 \\
CB(SAC) & 17,25 & $2,2 \mathrm{E}-4$ & 8,59 & 0,29 & $5,3 \mathrm{E}-3$ & 17,38 & 0,99
\end{tabular}

Fonte: Dados do autor.

\section{Isotermas de adsorção}

As isotermas de adsorção, $\mathrm{q}_{\mathrm{eq}}$ versus $\mathrm{C}_{\mathrm{eq}}$, estão apresentadas na Figura 4. Foi possível verificar um aumento na capacidade de adsorção de Cr para os resíduo modificados com $\mathrm{NaOH}$. Entretanto, as modificações com $\mathrm{NaOH}$ e ácido cítrico, não se mostraram muito eficientes, quando comparadas com o material in natura (Figura 4).

Fonte: Dados do autor. 
Figura 4 - Isotermas de adsorção do $\mathrm{Cr}$ (III) para $\mathrm{C}$, C-S, C-SAC, B, B-S, B-SAC, CB, CB-S, CB-SAC. Condições experimentais: $\mathrm{pH} 5$, tempo de agitação 500 min e temperatura $250 \mathrm{C}$.

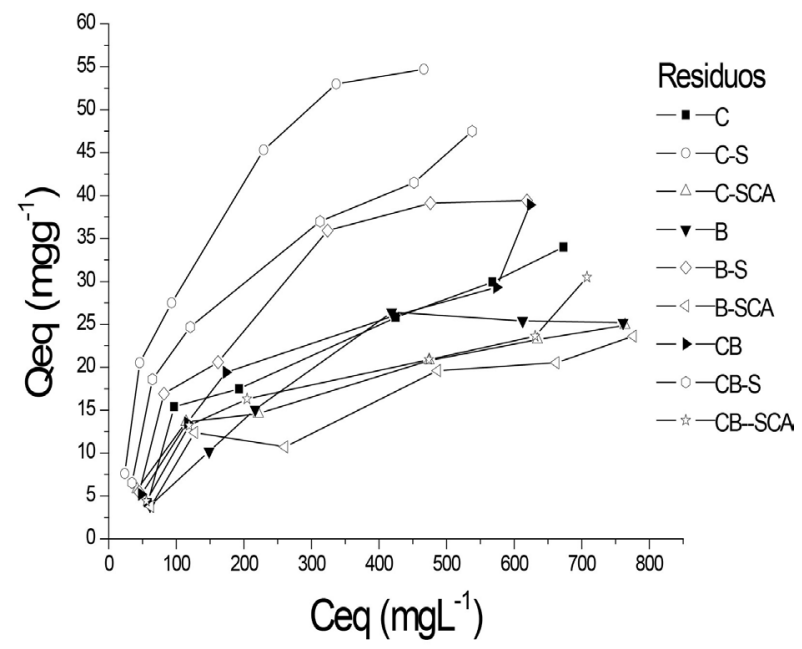

Fonte: Dados do autor.

Os dados obtidos das isotermas foram ajustados utilizando os modelos de Langmuir e de Freundlich, equações 4 e 5 , respectivamente.

$$
\frac{c_{e q}}{q_{e q}}=\frac{1}{q_{m} b}+\frac{c_{e q}}{q_{m}}
$$

Onde, $\mathrm{b}$ é uma constante que indica a intensidade da adsorção; qm é a medida da capacidade que indica a intensidade máxima de adsorção.

$$
\log q_{e q}=\log K_{f}+\left(\frac{1}{n}\right) \log C_{e q}
$$

Para esta equação, $K_{f}$ corresponde à constante de Freundlich $\left(\mathrm{K}_{\mathrm{f}}\right)$ e está relacionada com a capacidade de adsorção. A constante $\mathrm{n}$ relacionase com a intensidade de adsorção. Valores de $n$ na faixa $1<\mathrm{n}<10$ indicam adsorção favorável (PORPINO, 2009).

O modelo de Langmuir considera que apenas existe interação entre a molécula adsorvida e o adsorvente. Também assume que a interface sólida - líquida é homogênea e que a adsorção ocorre em uma única camada (monocamada) (HIEMENZ, 1986).

O modelo de Freundlich propõe que os íons metálicossão infinitamente acumulados na superfície do adsorvente, diferente de Langmuir, ocorre em multicamadas (YAMAMURA; YAMAURA, 2005). Este modelo ainda sugere que a energia de adsorção decresce logaritmicamente, à medida que a superfície do adsorvente vai se tornando coberta pelo soluto (ALCÂNTARA; CAMARGO, 2001).

Os valores dos parâmetros obtidos para cada modelo estão apresentados na Tabela 2. Pode-se verificar que o comportamento da adsorção para os resíduos de laranja aplica-se melhor ao modelo de Freundlich (valores de $\mathrm{r}^{2}$ mais próximo de 1), além disso, os valores de $\mathrm{n}$ foram maior do que 1 , o que indica que a adsorção ocorre de forma favorável.

Foi observado também que tanto a capacidade $\left(\mathrm{Q}_{\mathrm{m}}\right)$ quanto a intensidade de adsorção (b), tiveram os seus valores aumentados quando os resíduos de laranja receberam modificação química com $\mathrm{NaOH}$, indicando que ocorreu um aumento na afinidade do metal pelo adsorvente após o tratamento. A casca modificada com $\mathrm{NaOH}$ foi o resíduo com maior capacidade de retenção deste metal, sendo um promissor candidato a equipar filtros para a retenção deste elemento. Ao comparar os valores de Qm desse resíduo com outros estudos de biossorventes para o Cr (III) (Tabela 3), constatou-se uma maior eficiência deste adsorvente.

Embora a adsorção nos resíduos de laranja tenha seguido o modelo de Freundlich, a capacidade máxima de adsorção foi determinada pelo modelo de Langmuir, o qual tem sido largamente utilizado, pois pode ser empregado em altas concentrações dos íons (SODRÉ; LENZI; COSTA, 2001). 
Tabela 2 - Comparação dos parâmetros do modelo de Langmuir e de Freundlich, para adsorção de cromo (III) nos resíduos de laranja: C, C-S, C-SAC, B, B-S, B-SAC, CB, CB-S, CB-SAC.

\begin{tabular}{lcccccc}
\hline & \multicolumn{3}{c}{ Constantes de Langmuir } & \multicolumn{3}{c}{ Constantes de Freundlich } \\
Materiais & $\mathbf{Q}_{\mathbf{m}}$ & $\mathbf{b}$ & $\mathbf{r}^{\mathbf{2}}$ & $\mathbf{K}_{\mathrm{f}}$ & $\mathbf{n}$ & $\mathbf{r}^{\mathbf{2}}$ \\
\hline $\mathrm{C}$ & $\left(\mathrm{mg} \mathrm{g}^{-1}\right)$ & $\left(\mathrm{L} \mathrm{mg}^{-1}\right)$ & & $\left(\mathrm{mg} \mathrm{g}^{-1}\left(\mathrm{~L} \mathrm{mg}^{-1}\right)^{1 / n}\right)$ & \\
C(S) & 57,57 & $2,1 \mathrm{E}-3$ & 0.85 & 0,38 & 1,41 & 0,91 \\
C(SAC) & 75,64 & $6,3 \mathrm{E}-3$ & 0,97 & 1,04 & 1,48 & 0,98 \\
B & 29,92 & $5,8 \mathrm{E}-3$ & 0,98 & 0,89 & 1,94 & 0,98 \\
B(S) & 46,08 & $2,0 \mathrm{E}-3$ & 0,88 & 0,20 & 1,29 & 0,95 \\
B(SAC) & 62,26 & $3,4 \mathrm{E}-3$ & 0,89 & 0,59 & 1,43 & 0,90 \\
CB & 35,95 & $2,7 \mathrm{E}-3$ & 0,91 & 0,36 & 1,57 & 0,93 \\
CB(S) & 55,83 & $2,5 \mathrm{E}-3$ & 0,92 & 0,44 & 1,45 & 0,97 \\
CB(SAC) & 65,87 & $4,2 \mathrm{E}-3$ & 0,94 & 0,67 & 1,60 & 0,98 \\
\hline
\end{tabular}

Fonte: Dados do autor.

Tabela 3 - Comparação entre diferentes biossorventes para cromo (III).

\begin{tabular}{lcc}
\hline Adsorventes & $\begin{array}{c}\text { Qeq } \\
\left(\mathrm{mg} \mathrm{g}^{-1}\right)\end{array}$ & Referências \\
\hline Farelo de arroz & 0,13 & (Oliveira et al., 2005) \\
Nanotubo de carbono modificado & 0,50 & (Muataz et al., 2010) \\
Palha de sorgo & 9,35 & (Garcia-Reyes e Rangel-Mendez, 2010) \\
Palha de aveia & 12,10 & (Garcia-Reyes e Rangel-Mendez, 2010) \\
Resíduos de vinha & 12,45 & (Karaoğlu et al., 2010) \\
Bagaço agave & 28,72 & (Garcia-Reyes e Rangel-Mendez, 2010) \\
Carvão ativado & 30,00 & (Özacar e Sengil, 2003) \\
Casca $-\mathrm{NaOH}$ & 75,64 & Este estudo \\
\hline
\end{tabular}

Fonte: Dados do autor.

\section{Dessorção do cromo}

A reversibilidade da reação de adsorção é denominada dessorção. Este processo corresponde à retirada do metal do sítio de ligação da superfície do adsorvente. Um grande potencial de dessorção significa que o material pode ser reutilizado em novos processos de adsorção. Os valores obtidos para os resíduos de laranja estão expressos na Tabela 4.
Pode-se verificar que a porcentagem de metal dessorvido foi baixa, indicando que ocorre uma forte interação entre o cromo e o adsorvente, fato que corrobora com a cinética de pseudo-segunda ordem e a isoterma de Freundlich. Desta forma, estes resíduos não poderão ser reutilizados em um novo processo de adsorção. 
Tabela 4 - Valores de adsorção-dessorção do cromo (III) utilizando uma concentração inicial de 108 ppm, para os resíduos de laranja: C, C-S, C-SAC, B, B-S, B-SAC, CB, CB-S, CB-SAC.

\begin{tabular}{lccc}
\hline & $\begin{array}{c}\text { Concentração } \\
\text { Adsorvida }\end{array}$ & $\begin{array}{c}\text { Concentração } \\
\text { Dessorvida }\end{array}$ & $\begin{array}{c}\text { Dessorção } \\
\text { \% }\end{array}$ \\
\hline Materiais & $\left(\mathrm{mg} \mathrm{L}^{-1}\right)$ & $\left(\mathrm{mg} \mathrm{L}^{-1}\right)$ & \\
C & 55,70 & 13,69 & 24,58 \\
C(S) & 98,56 & 9,86 & 10,00 \\
C(SAC) & 93,66 & 24,16 & 25,79 \\
B & 53,01 & 12,20 & 23,01 \\
B(S) & 76,39 & 10,15 & 13,28 \\
B(SAC) & 69,42 & 17,46 & 25,15 \\
CB & 65,51 & 12,09 & 18,45 \\
CB(S) & 87,48 & 10,82 & 12,36 \\
CB(SAC) & 83,97 & 14,22 & 16,93 \\
\hline
\end{tabular}

Fonte: Dados do autor.

\section{Parâmetros Termodinâmicos}

Os valores dos parâmetros termodinâmicos, entalpia $(\Delta \mathrm{H})$, entropia $(\Delta \mathrm{S})$ e energia livre de Gibbs $(\Delta \mathrm{G})$, foram obtidos utilizando o gráfico de ln $\mathrm{Kd}$ versus $1 / \mathrm{T}$, em que pelo coeficiente angular da reta, calculou-se o valor de $\Delta \mathrm{H}\left(\frac{-\Delta H}{R}\right)$, e pelo coeficiente linear o valor de $\Delta \mathrm{S}\left(\frac{\Delta S}{R}\right)$, c o n for m e demonstrado na equação 6. Com os valores da entalpia e entropia determinados, pode-se então calcular os valores do $\Delta \mathrm{G}$ (equação 7 ), o qual se refere ao critério de espontaneidade da adsorção, ou seja, quanto mais negativo for o valor de $\Delta \mathrm{G}$, mais espontânea será a adsorção (ARAÚJO et al., 2009).

$$
\begin{aligned}
\ln k_{d} & =\left(\frac{\Delta S}{R}\right)-\left(\frac{\Delta H}{R}\right) \frac{1}{T} \\
\Delta G & =\Delta H-T \Delta S
\end{aligned}
$$

Onde:

$\mathrm{Kd}=$ coeficiente de distribuição do adsorbato $\left(\mathrm{Lg}^{-1}\right)$, corresponde a razão entre $\mathrm{q}_{\mathrm{eq}}$ e Ceq;

$\mathrm{T}=$ temperatura expressa em Kelvin $(\mathrm{K})$;

$\mathrm{R}=$ constante dos gases universal $\left(8.314 \mathrm{~J} \mathrm{~K}^{-1} \mathrm{~mol}^{-1}\right)$.
Após modificação química realizada nos resíduos de laranja verificou-se uma diminuição no valor de $\Delta \mathrm{G}$, tornando-os mais negativos em comparação com os resíduos in natura, deixando assim, a adsorção mais favorável energeticamente. Além disso, os menores valores de $\Delta \mathrm{G}$ foram para os resíduos modificados com $\mathrm{NaOH}$, corroborando com as maiores capacidades de adsorção $\left(\mathrm{Q}_{\mathrm{m}}\right)$, como foi apresentado na Tabela 2.

Tabela 5 - Parâmetros termodinâmicos obtidos a partir dos resultados de adsorção do cromo (III) nos resíduos de laranja: C, C-S, C-SAC, B, B-S, B-SAC, CB, CB-S,

\begin{tabular}{|c|c|c|c|c|}
\hline Materiais & $\mathrm{K}_{\mathrm{d}}$ & $\begin{array}{c}\Delta \mathrm{G} \\
\left(\mathrm{kJ} \mathrm{mol}{ }^{-1}\right)\end{array}$ & $\begin{array}{c}\Delta \mathrm{H} \\
\left(\mathrm{kJ} \mathrm{mol}^{-1}\right)\end{array}$ & $\begin{array}{c}\Delta \mathrm{S} \\
\left(\mathrm{kJ} \mathrm{mol}^{-1}\right)\end{array}$ \\
\hline $\mathrm{C}$ & 82,07 & $-36,64$ & $-5,52$ & 17,65 \\
\hline $\mathrm{C}(\mathrm{S})$ & 271,34 & $-46,58$ & $-6,34$ & 26,35 \\
\hline $\mathrm{C}(\mathrm{SAC})$ & 93,06 & $-37,69$ & $-13,91$ & $-8,66$ \\
\hline B & 58,73 & $-33,86$ & 9,97 & 65,18 \\
\hline $\mathrm{B}(\mathrm{S})$ & 145,95 & $-41,43$ & 11,74 & 79,81 \\
\hline $\mathrm{B}(\mathrm{SAC})$ & 74,88 & $-35,88$ & $-3,24$ & 24,04 \\
\hline $\mathrm{CB}$ & 77,32 & $-36,14$ & $-7,08$ & 13,73 \\
\hline $\mathrm{CB}(\mathrm{S})$ & 160,81 & $-42,23$ & 11,97 & 82,37 \\
\hline $\mathrm{CB}(\mathrm{SAC}$ & 98,13 & $-38,13$ & $-14,40$ & $-7,38$ \\
\hline
\end{tabular}
CB-SAC.

Fonte: Dados do autor.

Somente para B, B(S) e $\mathrm{CB}(\mathrm{S})$ os valores de entalpia foram positivos (Tabela 5), indicando que esta adsorção é de natureza endotérmica. Porém, os demais resíduos apresentaram valores negativos caracterizando um processo exotérmico. Para a entropia $(\Delta S)$, observou-se valores menores que zero para $\mathrm{C}(\mathrm{SAC})$ e $\mathrm{CB}(\mathrm{SAC})$, demonstrando que ocorreu uma certa ordenação do íon cromo (III) na superfície do adsorvente. No entanto, para os demais resíduos os valores foram positivos indicando, desta forma, um aumento da desordem na interface sólido-solução. 


\section{Conclusões}

As modificações químicas foram comprovadas utilizando espectroscopia na região do infravermelho, que indicou o aumento de sítios de adsorção. O tratamento químico proporcionou uma melhora nas propriedades adsorventes dos materiais estudados, tendo maior destaque a modificação apenas com $\mathrm{NaOH}$. Entre os resíduos de laranja, a casca apresentou uma maior capacidade de adsorver o íon cromo (III). A adsorção seguiu uma cinética de pseudo-segunda ordem e o modelo de adsorção de Freundlich, indicando que o material possui uma superfície heterogênea e uma forte interação adsorvente-adsorbato, evidenciada pelos baixos valores de dessorção do metal. Ainda, foi possível verificar, que além de aumentar as propriedades adsorventes, o sistema tornou-se mais espontâneo energeticamente após o tratamento químico, comprovado pela energia de Gibbs.

\section{Agradecimentos}

Nossos agradecimentos à Universidade Paranaense (UNIPAR) pelo apoio financeiro recebido e à Diretoria Executiva de Gestão da Pesquisa e da Pós-Graduação (DEGPP) pelo incentivo aos programas de iniciação científica (PIC e PIBIC) e ao mestrado em Biotecnologia Aplicado à Agricultura. A Fundação Araucária, CNPq e CAPES, pela bolsa concedida aos alunos.

\section{Referências}

ALCÂNTARA, M. A. K.; CAMARGO, O. A. Isotermas de adsorção de Freundlich para crômio (III) em latossolos. Scientia Agrícola, Piracicaba, v. 58, p. 567-572, 2001.

ANNADURAI, A.; JUANG, R. S.; LEE, D. J. Adsorption of heavy metals from water using banana and orange peels. Water Science and Technology, Oxford, v. 47, p. 185-190, 2003.

ARAÚJO, A. L. P.; SILVA, M. C. C.; GIMENES, M. L.; BARROS, M. A. S. D. Estudo termodinâmico de adsorção de Zn em argila bentonita bofe calcinada. Scientia Plena, Aracajú, v. 5, n. 12, p. 1-6, 2009.
BALDISSERA, B. L. Absorção/Adsorção de cádmio, cromo e chumbo por Agaricus blazei. 2007. Dissertação (Mestrado em Ciências Biológicas) - Instituto de Biociências do Campus de Rio Claro. Faculdade de Ciências Biológicas, Universidade Estadual Paulista, Rio Claro.

CONSELHO NACIONAL DO MEIO AMBIENTE. CONAMA. Resolução Conama $n^{\circ} 357$ de 17 abr. 2005. Disponível em: <http://www.mma.gov.br/port/conama/ legiano1.cfm? codlegitipo $=3 \& a n o=2005>$. Acesso em: 5 nov. 2010.

COSTA, C. A.; SCHNEIDER, I. A. H.; RÚBIO, J. Remoção de metais por subproduto de carvão. Saneamento ambiental, São Paulo, n. 59, p. 50-56, 1999.

DOĞAN, M.; ALKAN, M.; TURKYILMAZ, A.; ÖZDEMIR, Y. Kinetics and mechanism of removal of methylene blue by adsorption onto perlite. Journal of Hazardous Materials B, Amsterdam, v. 104, n. 1/3, p. 141-148, 2004.

SANTOS, V. C. G.; SOUZA, J. V. T. M.; TARLEY, C. R. T.; CAETANO, J.; DRAGUNSKI, D. C. Assessment of chemically modified sugarcane bagasse for lead adsorption from aqueous medium. Water Science and Technology, Oxford, v. 62, n. 2, p. 457-465, 2010.

SANTOS, V. C. G.; TARLEY, C. R. T.; CAETANO, J.; DRAGUNSKI, D. C. Copper ions adsorption from aqueous medium using the biosorbent sugarcane bagasse in natura and chemically modified. Water, Air, and Soil Pollution, Dordrecht, v. 216, n. 1/4, p. 351-359, 2011.

FENG, N.; GUO, X.; LIANG, S. Adsorption study of copper (II) by chemically modified orange peel. Journal of Hazardous Materials, Amsterdam, v. 164, p. 12861292, 2009.

GARCIA-REYES, R. B.; RANGEL-MENDEZ, J. R. Adsorption kinetics of chromium (III) ions on agrowaste materials. Bioresource Technology, Essex, v. 101, p. 8099-8108, 2010.

HIEMENZ, P. C. Principles of colloid and surface chemistry. 2. ed. New York: Marcel Dekker, 1986.

IMMICH, A. P. S. Remoção de corantes de efluentes têxteis utilizando folhas de Azadirachta indica como Adsorvente. 2006. Dissertação (Mestrado em Engenharia Química) - Universidade Federal de Santa Catarina, Florianópolis.

KARAOĞLU, M. H.; ZOR, S.; UGURLU, M. Biosorption of $\mathrm{Cr}$ (III) from solutions using vineyard pruning waste. Chemical Engineering Journal, Laussane, v. 159 , p. $98-106,2010$. 
KARNITZ, J. O.; GURGEL, L. V. A.; DE FREITAS, R. P.; GIL, L. F. Adsorption of $\mathrm{Cu}(\mathrm{II}), \mathrm{Cd}(\mathrm{II})$, and $\mathrm{Pb}$ (II) from aqueous single metal solutions by mercerized cellulose and mercerized sugarcane bagasse chemically modified with EDTA dianhydride (EDTAD). Carbohydrate Polymers, Barking, v. 77, p. 643-650, 2009.

KARNITZ, J. O.; GURGEL, L. V. A.; GIL, L. F. Removal of $\mathrm{Ca}$ (II) and $\mathrm{Mg}$ (II) from aqueous single metal solutions by mercerized cellulose and mercerized sugarcane bagasse grafted with EDTA dianhydride (EDTAD). Carbohydrate Polymers, Barking, v. 79, p. 184-191, 2010.

KURNIAWAN, T. A.; CHAN, G. Y. S.; LO, W. H.; BABEL, S. Comparisons of low-cost adsorbents for treating wastewaters laden with heavy metals. Science of the Total Environment, Amsterdam, v. 366, p. 409-426, 2006.

LIU, Y.; SUN, X.; LI, B. Adsorption of Hg2+ and Cd2+ by ethylenediamine modified peanut shells. Carbohydrate Polymers, Barking, v. 81, p. 335-339, 2010.

MARSHALL, W. E.; WARTELLE, L. H.; BOLER, D. E.; JOHNS, M. M.; TOLES, C. A. Enhanced metal adsorption by soybean hulls modified with nitric acid. Bioresource Technology, Essex, v. 69, p. 263-268, 1999.

MIMURA, A. M. S.; VIEIRA, T. V. A.; MARTELLI, P. B.; GORGULHO, H. F. Aplicação da casca de arroz na adsorção dos íons $\mathrm{Cu} 2+, \mathrm{Al} 3+, \mathrm{Ni2}+, \mathrm{Zn} 2+$. Química Nova, São Paulo, v. 33, n. 6, p. 1279-1284, 2010.

MUATAZ, A. A.; OMER, Y. B.; BASSAM, S. T.; ALAADIN, A. B.; MAZEN, K.; MAMDOUH, A.; MOHAMMED, F.; FARAJ, A. A. Removal of Chromium (III) from Water by Using Modified and Nonmodified Carbon Nanotubes. Journal of Nanomaterials, 2010. Disponível em: <http://www.hindawi.com/journals/ jnm/2010/232378/

NOELINE, B. F.; MANOHAR, D. M.; ANIRUDHAN, T. S. Kinetic and equilibrium modeling of lead (II) sorption from water and wastewater by polymerized banana stem in a batch reactor. Separation and Purification Technology, Monticello, NY, v. 45, p. 131-140, 2005.

OLIVEIRA, E. A.; MONTANHER, S. F.; ANDRADE, A. D.; NÓBREGA, J. A.; ROLlEMBERG, M. C. Equilibrium studies for the sorption of chromium and nickel from aqueous solutions using raw rice bran. Process Biochemistry, London, v. 40, p. 3485-3490, 2005.

ÖZCAN, A.; ÖZACAN, A. S.; TUNALI, S.; AKAR, T.; KIRAN, I. Determination of the equilibrium kinetic and thermodynamic parameters of adsorption of copper
(II) ions onto seeds of capsicum annuum. Journal of Hazardous Materials B, Amsterdam, v. 124, p. 200-208, 2005.

ÖZACAR, M.; SENGIL, I. A. Adsorption of reactive dyes on calcined alunite from aqueous solutions. Journal of Hazardous Materials, Amsterdam, v. 98, p. 211-224, 2003.

PAGNANELLI, F.; TRIFONI, M.; BEOLCHINI, F.; ESPÓSITO, A.; TORO, L.; VEGLIO, F. Equilibrium biosorption studies in single and multi-metal systems. Process Biochemistry, London, v. 37, n. 2, p. 115-124, 2001. Disponivel em: <http://www.ingentaconnect. com/content/els/00329592/2001/00000037/00000002/ art00180 - aff_1>. Acesso em: 5 nov. 2010.

PEHLIVAN, E.; ALTUN, T.; CETIN, S.; BHANGER, M. I. Lead sorption by waste biomass of hazelnut and almond shell. Journal of Hazardous Materials, Amsterdam, v. 167, p. 1203-1208, 2009.

PÉREZ-MARÍN, A. B.; ZAPATA, V. M.; ORTUÑO, J. F.; AGUILAR, M.; SÁEZ, J.; LORÉNS, M. Removal of cadmium from aqueous solutions by adsorption onto orange waste. Journal of Hazardous Materials, Amsterdam, B139, p. 122-131, 2007.

PORPINO, K. K. P. Biossorção de ferro (II) por casca de caranguejo Ucides Cordatus. 2009. Dissertação(Mestrado em Quimica) - Universidade Federal da Paraíba, João Pessoa. Disponível em: <http://www.quimica.ufpb.br/ posgrad/dissertacoes/Dissertacao_Karina_Karla .pdf>. Acesso em: 29 out. 2010.

RODRIGUES, R. F.; TREVEZOLI, R. L.; SANTOS, L. R. G.; LEÃO, V. A., BOTARO, V. R. Heavy metals sorption on treated wood sawdust. Engenharia Sanitária e Ambiental, Rio de Janeiro, v. 11, n. 1, p. 21-26, 2006.

SETHUNATHAN, N.; MEGHARAJ, M.; SMITH, L.; KAMALUDEEN, S. P. B.; AVUDAINAYAGAM, S.; NAIDU, R. Microbial role in the failure of natural attenuation of chromium(VI) in long-term tannery waste contaminated soil. Agriculture Ecosystems Environmet, Amsterdam, v. 105, p. 657-661, 2005.

SODRÉ, F. F.; LENZI, E.; COSTA, A. C. Applicability of adsorption models to the study of copper behaviour in clayey soils. Química Nova, São Paulo, v. 24, p. 324330, 2001.

SPINELLI, V. A.; LARANJEIRA, M. C. M.; FÁVERE, V. T.; KIMURA, I. Y. Cinética e equilíbrio de adsorção dos oxiânions Cr (VI), Mo (VI) e Se (VI) pelo sal de amônio quaternário de quitosana. Polímeros: Ciência e Tecnologia, São Carlos, v. 15, p. 218-223, 2005. 
TANA, G.; HONGYAN, Y.; YONG, L.; DAN, X. Removal of lead from aqueous solution with native and chemically modified corncobs. Journal of Hazardous Materials, Amesterdam, v. 174, p. 740-745, 2010.

YAMAMURA, A. P. G.; YAMAURA, M. Estudo da cinética e das isotermas de adsorção de U pelo bagaço de cana-de-açúcar. In: INTERNATIONAL NUCLEAR ATLANTIC CONFERENCE - INAC, Santos, 2005. CD-ROM.

YAO, Z.-Y.; QI, J.-H.; WANG, L.-H. Equilibrium, kinetic and thermodynamic studies on the biosorption of $\mathrm{Cu}$ (II) onto chestnut shell. Journal of Hazardous Materials, Amsterdam, v. 174, p. 137-143, 2010.

YUN, Y. S.; PARK, D.; PARK, J. M.; VOLESKY, B. Biosorption of trivalent chromium on the brown seaweed biomass. Environmental Science \& Technology, Easton, v. 35, p. 4353-4358, 2001.

Recebido em 10 Março 2011-Received on March 10, 2011.

Aceito em 21 Março, 2012 - Accepted on March 21, 2012. 
